\title{
Front Matter: Volume 6889
}

, "Front Matter: Volume 6889," Proc. SPIE 6889, Physics and Simulation of Optoelectronic Devices XVI, 688901 (10 March 2008); doi:

10.1117/12.791596

SPIE Event: Integrated Optoelectronic Devices 2008, 2008, San Jose, California, SPIE. United States 


\section{PROCEEDINGS OF SPIE}

\section{Physics and Simulation of Optoelectronic Devices XVI}

Marek Osiński

Fritz Henneberger

Keiichi Edamatsu

Editors

21-24 January 2008

San Jose, California, USA

Sponsored and Published by

SPIE

Volume 6889 
The papers included in this volume were part of the technical conference cited on the cover and title page. Papers were selected and subject to review by the editors and conference program committee. Some conference presentations may not be available for publication. The papers published in these proceedings reflect the work and thoughts of the authors and are published herein as submitted. The publisher is not responsible for the validity of the information or for any outcomes resulting from reliance thereon.

Please use the following format to cite material from this book:

Author(s), "Title of Paper," in Physics and Simulation of Optoelectronic Devices XVI, edited by Marek Osiński, Fritz Henneberger, Keiichi Edamatsu, Proceedings of SPIE Vol. 6889 (SPIE, Bellingham, WA, 2008) Article CID Number.

ISSN 0277-786X

ISBN 9780819470645

Published by

SPIE

P.O. Box 10, Bellingham, Washington $98227-0010$ USA

Telephone +1 3606763290 (Pacific Time) · Fax +1 3606471445

SPIE.org

Copyright (c) 2008, Society of Photo-Optical Instrumentation Engineers

Copying of material in this book for internal or personal use, or for the internal or personal use of specific clients, beyond the fair use provisions granted by the U.S. Copyright Law is authorized by SPIE subject to payment of copying fees. The Transactional Reporting Service base fee for this volume is $\$ 18.00$ per article (or portion thereof), which should be paid directly to the Copyright Clearance Center (CCC), 222 Rosewood Drive, Danvers, MA 01923. Payment may also be made electronically through CCC Online at copyright.com. Other copying for republication, resale, advertising or promotion, or any form of systematic or multiple reproduction of any material in this book is prohibited except with permission in writing from the publisher. The CCC fee code is 0277-786X/08/\$18.00.

Printed in the United States of America.

Publication of record for individual papers is online in the SPIE Digital Library.

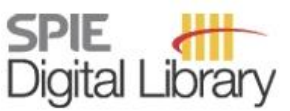

SPIEDigitalLibrary.org

Paper Numbering: Proceedings of SPIE follow an e-First publication model, with papers published first online and then in print and on CD-ROM. Papers are published as they are submitted and meet publication criteria. A unique, consistent, permanent citation identifier (CID) number is assigned to each article at the time of the first publication. Utilization of CIDs allows articles to be fully citable as soon they are published online, and connects the same identifier to all online, print, and electronic versions of the publication. SPIE uses a six-digit CID article numbering system in which:

- The first four digits correspond to the SPIE volume number.

- The last two digits indicate publication order within the volume using a Base 36 numbering system employing both numerals and letters. These two-number sets start with 00, 01, 02, 03, 04, 05, $06,07,08,09,0 \mathrm{~A}, 0 \mathrm{~B} \ldots \mathrm{OZ}$, followed by $10-1 \mathrm{Z}, 20-2 \mathrm{Z}$, etc.

The CID number appears on each page of the manuscript. The complete citation is used on the first page, and an abbreviated version on subsequent pages. Numbers in the index correspond to the last two digits of the six-digit CID number. 


\section{Contents}

vii Conference Committee

WIDE-BANDGAP LASERS AND LEDS

688903 Optical gain and spontaneous emission of strain-compensated InGaN-AIGaN quantum wells including carrier screening effect [6889-02]

H. Zhao, R. A. Arif, Y. K. Ee, N. Tansu, Lehigh Univ. (USA)

688904 Thick InGaN growth on several crystal planes of ZnO substrate by metalorganic vapor phase epitaxy [6889-03]

Y. Kawai, S. Ohsuka, M. Iwaya, S. Kamiyama, H. Amano, I. Akasaki, Meijo Univ. (Japan)

688905 Effect of interface polarization charge on the performance of nitride semiconductor light emitting diodes [6889-04]

H. Shen, M. L. Reed, E. D. Readinger, M. Wraback, U.S. Army Research Lab. (USA)

\section{PHOTOVOLTAIC DEVICES}

688906 Present and future of super high efficiency multi-junction solar cells (Invited Paper) [6889-05]

M. Yamaguchi, Toyota Technological Institute (Japan); T. Takamoto, Sharp Corp. (Japan); K. Araki, Daido Steel Corp. (Japan)

\section{NONLINEAR DYNAMICS AND CHAOS IN SEMICONDUCTOR LASERS}

688909 All-optical noninvasive control of semiconductor lasers (Invited Paper) [6889-08]

S. Schikora, H.-J. Wünsche, F. Henneberger, Humboldt Univ. of Berlin (Germany)

6889 OA Semiconductor laser subject to intense feedback with variably rotated polarization (Invited Paper) [6889-09]

N. Gross, Z. Shotan, T. Galfsky, L. Khaykovich, Bar-llan Univ. (Israel)

6889 OB Nonlinear dynamics and chaotic behavior observed in laterally coupled diode lasers [6889-10]

R. Santos, H. Lamela, Univ. Carlos III de Madrid (Spain)

PHYSICS OF LOW-DIMENSIONAL STRUCTURES AND DEVICES

6889 OD Nonlinear excitations of semiconductor quantum wells with intense terahertz fields (Invited Paper) [6889-12]

J. T. Steiner, M. Kira, S. W. Koch, Philipps-Univ. Marburg (Germany) 
6889 OE Temperature dependence of radiative and Auger losses in quantum well lasers [6889-13] J. Hader, J. V. Moloney, Nonlinear Control Strategies Inc. (USA) and Optical Sciences Ctr., Univ. of Arizona (USA); S. W. Koch, Philipps-Univ. Marburg (Germany)

\section{PHYSICS AND SIMULATION OF VCSELS AND DISK LASERS}

$6889 \mathrm{OH} \quad$ Ultra high-speed electro-optically modulated VCSELs: modeling and experimental results (Invited Paper) [6889-16]

V. A. Shchukin, N. N. Ledentsov, VI Systems GmbH (Germany), Technische Univ. Berlin (Germany), and Abraham loffe Physical Technical Institute (Russia); J. A. Lott, H. Quast, VI Systems GmbH (Germany); F. Hopfer, Technische Univ. Berlin (Germany); L. Ya. Karachinsky, Technische Univ. Berlin (Germany) and Abraham loffe Physical Technical Institute (Russia); M. Kuntz, P. Moser, A. Mutig, A. Strittmatter, Technische Univ. Berlin (Germany);

V. P. Kalosha, Univ. of Ottawa (Canada); D. Bimberg, Technische Univ. Berlin (Germany)

6889 Ol A robust electro-optothermal coupling scheme for semiconductor laser simulation [6889-17]

R. Santschi, Synopsys Switzerland, LLC (Switzerland); H. Meier, ETH Zürich (Switzerland);

S. Odermatt, Synopsys Switzerland, LLC (Switzerland)

\section{PHYSICS OF QUANTUM DOT/DASH LASERS AND AMPLIFIERS}

6889 OK Compact quantum-dot-based ultrafast lasers (Invited Paper) [6889-47]

W. Sibbett, Univ. of St. Andrews (United Kingdom); E. U. Rafailov, Univ. of Dundee (United Kingdom)

6889 OL Recent developments of InP-based quantum dashes for directly modulated lasers and semiconductor optical amplifiers (Invited Paper) [6889-20]

F. Lelarge, R. Brenot, B. Rousseau, F. Martin, Alcatel-Thales III-V Lab. (France); G. Patriarche, Lab. for Photonics and Nanostructures (France); F. Poingt, L. LeGouezigou,

O. Le Govezigou, C. Dernazaretian, E. Derouin, O. Drisse, F. Pommereau, A. Accard,

M. Caligaro, D. Make, J.-G. Provost, P. Resneau, B. Dagens, F. van-Dijk, M. Krakowski,

G. H. Duan, Alcatel-Thales III-V Lab. (France)

6889 OM Giant nonlinear gain coefficient of an InAs/AIGalnAs quantum dot laser [6889-21]

L. F. Lester, Ctr. for High Technology Materials, Univ. of New Mexico (USA); N. B. Terry, Air

Force Research Lab. (USA); A. J. Moscho, Ctr. for High Technology Materials, Univ. of New Mexico (USA); M. L. Fanto, Air Force Research Lab. (USA); N. A. Naderi, Y. Li, Ctr. for High Technology Materials, Univ. of New Mexico (USA); V. Kovanis, Air Force Research Lab. (USA)

\section{PHOTONICS WITH SINGLE-QUANTUM-DOT DEVICES}

6889 OS Resonantly excited emissions from a GaAs quantum dot and an exciton dipole moment [6889-24]

K. Kuroda, National Institute for Materials Science (Japan); T. Kuroda, National Institute for Materials Science (Japan) and PRESTO, Japan Science and Technology Agency (Japan); K. Sakoda, National Institute for Materials Science (Japan) and Univ. of Tsukuba (Japan); G. Kido, N. Koguchi, National Institute for Materials Science (Japan) 
6889 OU Dipole lasing stimulated by nanoantenna (Invited Paper) [6889-25]

I. E. Protsenko, A. V. Uskov, K. E. Krotova, P.N. Lebedev Physical Institute (Russia) and

Advanced Energy Technology Ltd. (Russia); E. P. O'Reilly, Tyndall National Institute (Ireland)

PHOTONIC CRYSTAL DEVICES

6889 OW High-power one-, two-, and three-dimensional photonic crystal edge-emitting laser diodes for ultra-high brightness applications (Invited Paper) [6889-27]

N. Yu. Gordeev, M. V. Maximov, Y. M. Shernyakov, I. I. Novikov, Abraham loffe PhysicoTechnical Institute (Russia); L. Ya. Karachinsky, Abraham loffe Physico-Technical Institute (Russia) and Technische Univ. Berlin (Germany); V. A. Shchukin, T. Kettler, K. Posilovic,

N. N. Ledentsov, D. Bimberg, Technische Univ. Berlin (Germany); R. Duboc, A. Sharon,

D. B. Arbiv, U. Ben-Ami, PBC Lasers Ltd. (Israel)

\section{PHOTONIC CRYSTAL NANOCAVITY DEVICES}

688910 Ultrafast photonic crystal nanocavity lasers and optical switches (Invited Paper) [6889-32] I. Fushman, D. Englund, Stanford Univ. (USA); H. Altug, Boston Univ. (USA); B. Ellis, A. Faraon, J. Vučković, Stanford Univ. (USA)

688911 Physics and simulation of photonic crystal Purcell light emitters (Invited Paper) [6889-33] B. Witzigmann, F. Römer, ETH Zürich (Switzerland)

\section{MID-INFRARED DEVICES}

688912 Thermal analysis of mid-infrared quantum-cascade lasers (Invited Paper) [6889-40]

C. A. Evans, D. Indjin, Z. Ikonić, P. Harrison, Univ. of Leeds (United Kingdom)

688913 Strain-compensated AIAs-InGaAs quantum-cascade lasers with emission wavelength 3-5 $\mu \mathrm{m}$ (Invited Paper) [6889-39]

W. T. Masselink, M. P. Semtsiv, M. Wienold, M. Chashnikova, I. Bayrakli, M. Klinkmüller, Humboldt-Univ. zu Berlin (Germany)

$688914 \mathrm{Mid} / \mathrm{far}$-infrared photodetection based on resonant frequency up-conversion in coupled quantum wells [6889-41]

A. K. Wójcik, F. Xie, V. R. Chaganti, A. Belyanin, Texas A\&M Univ. (USA); J. Kono, Rice Univ. (USA)

\section{PHYSICS OF RING LASERS AND DIODE LASER FREQUENCY STABILIZATION}

688915 Monolithic InP-based passively modelocked semiconductor ring lasers at $1.5 \mu \mathrm{m}$ (Invited Paper) [6889-35]

E. Bente, M. Heck, Y. Barbarin, S. Anantathanasarn, R. Nötzel, M. Smit, Technische Univ.

Eindhoven (Netherlands) 
688916 Highly unidirectional Y-junction S-section ring lasers [6889-36]

N. J. Withers, O. K. Qassim, G. A. Smolyakov, M. Osiński, Ctr. for High Technology Materials, The Univ. of New Mexico (USA)

688917 Semiconductor laser frequency-stabilization: influence of multi-position temperature controls [6889-37]

M. Yanagisawa, K. Nakano, S. Maehara, T. Sato, M. Ohkawa, T. Maruyama, Niigata Univ. (Japan); S. Kawamura, National Astronomical Observatory (Japan)

688918 Frequency stabilization of an external cavity diode laser: countermeasures against atmospheric temperature variations [6889-38]

Y. Minabe, K. Doi, T. Sato, T. Maruyama, M. Ohkawa, Niigata Univ. (Japan); T. Tsubokawa, Mizusawa Astrogeodynamic Observatory, National Astronomical Observatory of Japan (Japan)

\section{POSTER SESSION}

688919 All-optical signal processing in photonic crystals covered with fast nonlinear materials [6889-30]

E. Ya. Glushko, Institute of Semiconductor Physics (Ukraine); A. E. Glushko, Institute of Semiconductor Physics (Ukraine) and Univ. of Leoben (Austria); V. N. Evteev,

A. N. Stepanyuk, Krivoy Rog State Pedagogical Univ. (Ukraine)

6889 1C A novel acousto-optic tunable filter for use in hyperspectral imaging systems [6889-46] C. Stedham, Gooch \& Housego UK Ltd. (United Kingdom) and Heriot-Watt Univ. (United Kingdom); M. Draper, J. Ward, Gooch \& Housego UK Ltd. (United Kingdom); E. Wachman, ChromoDynamics Inc. (USA); C. Pannell, Optronic Labs., Inc. (USA)

Author Index 


\title{
Conference Committee
}

\author{
Symposium Chair
}

Ali Adibi, Georgia Institute of Technology (USA)

Symposium Cochair

James G. Grote, Air Force Research Laboratory (USA)

Program Track Chair

James G. Grote, Air Force Research Laboratory (USA)

Conference Chairs

Marek Osiński, Center for High Technology Materials, The University of New Mexico (USA)

Fritz Henneberger, Humboldt-Universität zu Berlin (Germany)

Keiichi Edamatsu, Tohoku University (Japan)

Program Committee

Michael J. Adams, University of Essex (United Kingdom)

Hiroshi Amano, Meijo University (Japan)

Yasuhiko Arakawa, The University of Tokyo (Japan)

Toshihiko Baba, Yokohama National University (Japan)

Peter Blood, Cardiff University (United Kingdom)

Weng W. Chow, Sandia National Laboratory (USA)

Shun Lien Chuang, University of Illinois at Urbana-Champaign (USA)

Silvano Donati, Università degli Studi di Pavia (Italy)

Athanasios Gavrielides, Air Force Research Laboratory (USA)

Stephan W. Koch, Philipps-Universität Marburg (Germany)

Fumio Koyama, Tokyo Institute of Technology (Japan)

Nikolai N. Ledentsov, Technische Universität Berlin (Germany)

Luigi A. Lugiato, Università degli Studi dell'Insubria (Italy)

Cun-Zheng Ning, Arizona State University (USA)

Joachim Piprek, NUSOD Institute (USA)

Paul Hongen Shen, Army Research Laboratory (USA)

Claude Weisbuch, Ecole Polytechnique (France)

\section{Session Chairs}

Wide-Bandgap Lasers and LEDs

Marek Osiński, Center for High Technology Materials, The University of New Mexico (USA) 
Photovoltaic Devices

Paul Hongen Shen, Army Research Laboratory (USA)

Nonlinear Dynamics and Chaos in Semiconductor Lasers

Bernd Witzigmann, ETH Zürich (Switzerland)

Physics of Low-Dimensional Structures and Devices

Yasuhiko Arakawa, The University of Tokyo (Japan)

Physics and Simulation of VCSELs and Disk Lasers

Shun Lien Chuang, University of Illinois at Urbana-Champaign (USA)

Physics of Quantum Dot/Dash Lasers and Amplifiers

Luke E. Lester, Center for High Technology Materials, The University of New Mexico (USA)

Surface Plasmon Devices

Cun-Zheng Ning, Arizona State University (USA)

Photonics with Single-Quantum-Dot Devices

Fritz Henneberger, Humboldt-Universität zu Berlin (Germany)

Nanoscale Devices

Nikolai N. Ledentsov, Technische Universität Berlin (Germany)

Photonic Crystal Devices

Ilya Fushman, Stanford University (USA)

Photonic Crystal Nanocavity Devices

Claude Weisbuch, University of California, Santa Barbara (USA)

Mid-Infrared Devices

Takashi Sato, Niigata University (Japan)

Physics of Ring Lasers and Diode Laser Frequency Stabilization Vitaly Shchukin, V.I. Systems GmbH (Germany) 